\title{
Towards functional flows for hierarchical models
}

\author{
Daniel F. Litim* \\ Department of Physcis and Astronomy, University of Sussex, Brighton, BN1 9QH, United Kingdom \\ Theory Group, Physics Division, CERN, CH-1211 Geneva 23, Switzerland
}

(Received 25 April 2007; published 2 November 2007)

\begin{abstract}
The recursion relations of hierarchical models are studied and contrasted with functional renormalization group equations in corresponding approximations. The formalisms are compared quantitatively for the Ising universality class, where the spectrum of universal eigenvalues at criticality is studied. A significant correlation amongst scaling exponents is pointed out and analyzed in view of an underlying optimization. Continuous functional flows are provided which match with high accuracy all known scaling exponents from Dyson's hierarchical model for discrete block-spin transformations. Implications of the results are discussed.
\end{abstract}

DOI: 10.1103/PhysRevD.76.105001

\section{INTRODUCTION}

Renormalization group methods [1], and, in particular, Wilson's renormalization group [2], play an important role in the study of physical systems at strong coupling and/or large correlations lengths. Differential implementations of Wilson's idea [3-10] rely on an appropriately-introduced momentum cutoff leading to flow equations for running couplings and $N$-point functions, which can be studied with a large variety of analytical and numerical methods. Numerical stability and reliability in the results is ensured through powerful control and optimization techniques [1114]. A different implementation of Wilson's idea is realised in hierarchical models of lattice scalar theories [15-19]. Hierarchical renormalization group transformations are often discrete rather than continuous. Here, sophisticated numerical methods have been developed to extract the relevant physics, most notably for high-accuracy studies of scaling exponents for scalar models at criticality [2022] and related theories (see [19] and references therein).

Given the close similarity of the underlying principles, it is natural to ask whether Wilsonian (functional) flows can be linked explicitly, and on a fundamental level, to hierarchical models. If so, this link would provide a number of benefits. It will make powerful functional and numerical methods available to the study of hierarchical models. Vice versa, the numerical tools for hierarchical models could be employed for functional flows in specific approximations. Furthermore, an explicit link may lead to a path integral representation of hierarchical models, allowing for systematic improvements beyond a standard kinetic term. Finally, well-developed optimization techniques for functional flows could be taken over for hierarchical models as well.

In the limit of continuous hierarchical block-spin transformations, an explicit link between Dyson's hierarchical model $[15,17]$ and the Wilson-Polchinski flow [23] in the local potential approximation has been established long

\footnotetext{
*D.Litim@sussex.ac.uk Daniel.Litim@cern.ch
}

PACS numbers: 05.10.Cc, 11.10.Hi, 11.10.Kk, 64.60.Fr

ago by Felder [24]. Following a conjecture of [13], this link has been extended $[25,26]$ to include optimised versions $[11,12]$ of Wetterich's flow for the effective average action [27]. These interrelations have recently been backed-up by extensive numerical studies of critical potentials and scaling exponents to high accuracy from either formalism [28].

In this paper, we evaluate the more general case and ask whether hierarchical models for discrete block-spin transformations are linked to functional flows with continuous renormalization group transformations. We first contrast the basic setups for functional flows (Sec. II), background field flows (Sec. III), and hierarchical models (Sec. IV). At a Wilson-Fisher fixed point, underlying similarities and differences are worked out and compared for the leading scaling exponent (Sec. V). An extensive numerical study of the eigenvalue spectrum of the Ising universality class from functional flows is performed (Sec. VI). A strong correlation of scaling exponents is established and analyzed (Sec. VII). It is shown that specific functional flows match the leading and subleading scaling exponent from Dyson's hierarchical models for discrete transformation parameter to high accuracy (Sec. VIII). We close with a discussion of the results and further implications (Sec. IX).

\section{FUNCTIONAL FLOWS}

Wilsonian (functional) flows integrate out quantum fluctuations within a path integral representation of quantum field theory. In their simplest form, they are generated through a cutoff term quadratic in the field added to the Schwinger functional, where the (classical) action is replaced by $S \rightarrow S+\Delta S_{k} \quad$ and $\quad \Delta S_{k} \sim \int d q \phi(q) \times$ $R_{k}\left(q^{2}\right) \phi(-q)$. The infrared momentum cutoff $R_{k}\left(q^{2}\right)$ ensures that the propagation of small momentum modes $q^{2} \ll k^{2}$ is suppressed, while the large momentum modes $q^{2} \gg k^{2}$ remain unaffected. Under an infinitesimal change in the Wilsonian (infrared) cutoff scale $k$, the effective action $\Gamma_{k}$ changes according to its functional flow, which reads $(t=\ln k)$

$$
\partial_{t} \Gamma_{k}=\frac{1}{2} \operatorname{Tr}\left(\Gamma_{k}^{(2)}+R_{k}\right)^{-1} \partial_{t} R_{k}
$$


in the form put forward by Wetterich [27]. The trace denotes a momentum integration and a summation over fields. The factor $\partial_{t} R_{k}$ in the integrand is peaked in the vicinity of $q^{2} \approx k^{2}$. The cutoff function $R_{k}$ obeys $R_{k}\left(q^{2}\right) \rightarrow 0$ as $k^{2} / q^{2} \rightarrow 0, R_{k}\left(q^{2}\right)>0$ as $q^{2} / k^{2} \rightarrow 0$, and $R_{k}\left(q^{2}\right) \rightarrow \infty$ as $k \rightarrow \Lambda$, and can be chosen freely elsewise, e.g. [11]. It ensures that the flow is well-defined, thereby interpolating between an initial action $S$ at $k=\Lambda$ in the ultraviolet (UV) and the full quantum effective action $\Gamma \equiv$ $\Gamma_{k=0}$ in the infrared $k \rightarrow 0$.

In addition to providing a momentum cutoff, the function $R_{k}\left(q^{2}\right)$ also controls the stability and convergence of subsequent expansions [11-13,29]. Therefore, it is possible to identify optimized momentum cutoffs - within given systematic expansions - which improve the physical result $[11,12,30]$. The construction of optimized cutoffs [11-14] is central to extract reliable results also in more complex theories including e.g. QCD [31], quantum gravity [32], thermal physics [5,6,33], and critical phenomena $[28,30,34]$.

Below, we are interested in $3 d$ scalar theories at criticality, where we can sent the ultraviolet scale $\Lambda \rightarrow \infty$. To leading order in the derivative expansion, the effective action reads $\Gamma_{k}=\int d^{3} x\left[\frac{1}{2} \partial_{\mu} \phi \partial_{\mu} \phi+U_{k}(\bar{\rho})\right]$ and $\bar{\rho}=$ $\frac{1}{2} \phi^{2}$. Introducing $r(y)=R_{k}\left(q^{2}\right) / q^{2}$ with $y=q^{2} / k^{2}$, we find

$$
\partial_{t} u=-3 u+\rho u^{\prime}+\int_{0}^{\infty} d y \frac{-y^{3 / 2} r^{\prime}(y)}{y(1+r)+u^{\prime}+2 \rho u^{\prime \prime}}
$$

with $u(\rho)=U_{k}(\bar{\rho}) / k^{3}$ and $\rho=\bar{\rho} / k$. An irrelevant constant originating from the angular integration has been rescaled into the potential and the fields. For the optimal cutoff $R_{\mathrm{opt}}=\left(k^{2}-q^{2}\right) \theta\left(k^{2}-q^{2}\right) \quad$ with $\quad r_{\mathrm{opt}}=(1 / y-$ 1) $\theta(1-y)$, the flow reads [12]

$$
\partial_{t} u=-3 u+\rho u^{\prime}+\frac{1}{1+u^{\prime}+2 \rho u^{\prime \prime}}
$$

after an additional rescaling. This flow is integrated analytically in the limit of a large number of scalar fields [35]. We note that the universal content of the flow (3) is equivalent to the Wilson-Polchinski flow in the local potential approximation $[13,25,26]$.

\section{BACKGROUND FIELD FLOWS}

A different form of the flow (1) is obtained for momentum cutoffs which depend additionally on a background field $\bar{\phi}$. Background fields are most commonly used for the study of gauge theories [36], see [32,37,38] for applications. They have also been employed for a path integral derivation of (generalized) proper-time flows [39,40].

In the presence of background fields, the functional $\Gamma_{k}[\phi]$ turns into a functional of both fields, $\Gamma_{k}[\phi, \bar{\phi}]$. In order to maintain the one-loop exactness of (1), the momentum cutoff can only depend on the background field, but not on the propagating field. Following [39], we introduce $x=\Gamma^{(2,0)}[\phi, \phi]$ and $\bar{x}=x[\phi=\bar{\phi}]$, where $\Gamma_{k}^{(n, m)}[\phi, \bar{\phi}] \equiv \delta^{n} \delta^{m} \Gamma_{k} / \delta \phi^{n} \delta \bar{\phi}^{m}$. We chose momentum cutoffs of the form $R_{k}\left(q^{2}\right) \rightarrow \bar{x} r[\bar{x}]$, which depend now on the background field. Here, the regulator cuts off both large momentum modes $q^{2} \gg k^{2}$ and large field amplitudes with $\Gamma_{k}^{(2,0)} \gg k^{2}$. The full advantage of background fields becomes visible once they are identified with the physical mean, leading to the functional $\Gamma_{k}[\phi, \bar{\phi}=\phi] \rightarrow \Gamma_{k}[\phi]$. The resulting flow is closed provided $\Gamma_{k}^{(2)}[\phi]=$ $\Gamma_{k}^{(2,0)}[\phi, \phi]$. For scalars, this relation becomes exact in the infrared limit studied below (for gauge fields, see [38]). Using the momentum cutoffs [39]

$$
r_{\mathrm{PT}, m}[x]=\exp \left(\frac{1}{m}\left(\frac{m k^{2}}{x}\right)^{m}{ }_{2} F_{1}\left[m, m ; m+1 ;-\frac{m k^{2}}{x}\right]\right)-1,
$$

we are lead to the background field flow

$$
\begin{aligned}
\partial_{t} \Gamma_{k}= & \operatorname{Tr}\left(\frac{k^{2}}{k^{2}+x / m}\right)^{m}+\frac{1}{2} \operatorname{Tr}\left[\frac{r_{\mathrm{PT}, m}}{x\left(1+r_{\mathrm{PT}, m}\right)}\right. \\
& \left.-\left(\frac{k^{2}}{k^{2}+x / m}\right)^{m} \frac{1}{x}\right] \partial_{t} x .
\end{aligned}
$$

If the term $\sim \partial_{t} x$ on the right-hand side is droppedmeaning that additional flow terms originating from the implicit scale dependence in the momentum cutoff are neglected over the leading term - the flow (4) reduces to the proper-time flow of Liao [41]. A general proper-time flow is a linear combination of the first term in (4) for various $m$ [40]; see [42,43] for applications.

Next, we specialize to the proper-time approximation to leading order in the derivative expansion. The flow equation for the effective potential takes the very simple form

$$
\partial_{t} u=-3 u+\rho u^{\prime}+\frac{1}{\left(m+u^{\prime}+2 \rho u^{\prime \prime}\right)^{m-3 / 2}},
$$

where $m$ parametrizes the momentum cutoff, and an irrelevant constant factor has been rescaled into the potential and the fields. For $m \in\left[1, \frac{5}{2}\right]$, the flow (5) is mapped onto the flow (2) [39]. At $m=\frac{5}{2}$, the flow (5) is equivalent to (3), modulo a trivial rescaling. As a final remark, we note that this proper-time flow is also obtained from linear combinations of higher scale-derivatives of Callan-Szymanzik flows, without relying on background fields [40]. In this representation, the approximation leading to (5) consists in the neglect of higher order flow terms $\sim \partial_{t}^{n} \Gamma_{k}^{(2)}$.

\section{HIERARCHICAL MODELS}

Several hierarchical models for an effective potential $v(\varphi)$ of a lattice scalar field have been introduced in the literature [15-17] (see also [19]). The hierarchical trans- 
formation laws relate the potential $v(\varphi)$ at momentum scale $k / \ell$ with an average in field space over $v(\varphi)$ at momentum scale $k$, where $\ell \geq 1$ is the renormalization group step parameter. We restrict ourselves to the threedimensional case; the generalization to arbitrary dimensions is straightforward.

In Dyson's model [15,17], the renormalization group step $k \rightarrow k / \ell$ for the potential is expressed as

$$
e^{-v_{k / \ell}(\varphi)}=\int_{-\infty}^{+\infty} d \xi \mu_{\ell}(\xi) e^{-\ell^{3} v_{k}\left(\ell^{-1 / 2} \varphi+\xi\right)} .
$$

The details of the averaging procedure are encoded in the measure factor $\mu_{\ell}(\xi)$, in the $\xi$-dependence of the potential on the right-hand side of (6), and in the choice for the decimation parameter $\ell$. As is evident from (6), a decimation parameter $\ell=2^{1 / 3}$-employed for most numerical studies [19-22] — corresponds to a volume decimation of $\ell^{3}=2$ at each iteration. For Dyson's model, the measure is chosen as $\mu_{\ell}(\xi)=(\pi \sigma(\ell))^{-1 / 2} \exp \left(-\xi^{2} / \sigma(\ell)\right)$ [19], where we require $\sigma(\ell)>0$ for $\ell \neq 1$, and $\sigma(1)=0$ with $\sigma^{\prime}(1) \neq 0$. A standard choice is $\sigma(\ell)=2(\ell-1)$ [24]. By definition, (6) describes a flow towards the infrared for decimation parameters $\ell \geq 1$. For $\ell \rightarrow 1$, the hierarchical transformation (6) becomes continuous and the measure factor turns into a $\delta$-function $\mu_{\ell \rightarrow 1}(\xi) \rightarrow \delta(\xi)$. Performing $-\ell \partial_{\ell}(6)$, which is equivalent to $k \partial_{k}(6)$, we arrive at a differential flow equation for the effective potential [24]

$$
\partial_{t} v=-3 v+\frac{1}{2} \varphi v^{\prime}-v^{\prime \prime}+\left(v^{\prime}\right)^{2}
$$

where an irrelevant factor is rescaled into the fields and the potential; $t=\ln k$. The interaction terms in (7) originate from the scale-derivative of the measure $-\ell \partial_{\ell} \mu_{\ell}(\xi)$, which reads $\frac{1}{4} \sigma^{\prime}(1) \delta^{\prime \prime}(\xi)$ in the limit $\ell \rightarrow 1$. This highlights the relevance of the measure factor in hierarchical models. Our normalization corresponds to the choice $\sigma^{\prime}(1)=4$ to match with [28]. The limit (7) is independent of $\sigma$, but at $\ell \neq 1$, we expect that scaling solutions and exponents from (6) depend on it. Equation (7) is the wellknown Wilson-Polchinski flow $[2,23]$. We therefore conclude that the potential in (7) is related to the potential in (3) by a Legendre transformation [26,28].

A different version of a hierarchical model has been introduced by Wilson [16]. Here, the recursion relation is written as

$e^{-v_{k / \ell}(\varphi)}=\int_{-\infty}^{+\infty} d \xi \mu_{\ell}(\xi) e^{-(1 / 2) \ell^{3}\left[v_{k}\left(\ell^{-1 / 2} \varphi+\xi\right)+v_{k}\left(\ell^{-1 / 2} \varphi-\xi\right)\right]}$.

In Wilson's original model, the $\xi$-dependence of the measure is $\mu_{\ell}(\xi)=N_{\ell} \exp \left(-\xi^{2}\right)$, where the normalization factor $N_{\ell}$ is $\xi$-independent [19]. The measure factor is different from the one in Dyson's model, because the Gaussian width is $\ell$-independent. If instead we employ the measure of Dyson's model, the limit $\ell \rightarrow 1$ can be performed analytically. ${ }^{1}$ Up to a trivial rescaling, we find

$$
\partial_{t} v=-3 v+\frac{1}{2} \varphi v^{\prime}-v^{\prime \prime} .
$$

In contrast to the Wilson-Polchinski flow (7), the nonlinear term $\left(v^{\prime}\right)^{2}$ is absent. This comes about because the integrand of (8) - as opposed to the integrand of (6) - is manifestly symmetric under $\xi \rightarrow-\xi$. Numerical evaluations of (8) have been reported in [16,44]. For other representations of hierarchical models we refer to [19] and references therein.

\section{MATCHING HIERARCHICAL MODELS}

In order to match hierarchical models by functional flows, we have to detail the scheme dependences of physical observables in either formalism. In the functional RG framework, the fully integrated flow is independent of the momentum cutoff $R_{k}\left(q^{2}\right)$ chosen for the integration. Scheme dependences, which enter as a consequence of truncations of $\Gamma_{k}[\phi]$, have been discussed extensively in the literature $[11-13,29,45]$. Their origin is easily understood. Since the momentum cutoff $R$ in (1) couples to all operators in the theory, the missing back-coupling of operators neglected in a given truncation can result in a spurious dependence of physical observables evaluated either from $\Gamma_{0}[\phi]$, or from a fixed point solution $\Gamma_{*}[\phi]$. The scheme dependence is reduced by identifying those momentum cutoffs, which, in a given truncation, lead to an improved convergence and stability of the flow.

In Fig. 1, we discuss the scheme dependence quantitatively for the leading order scaling exponent $\nu$ at a fixed point of the $3 d$ Ising universality class [29]. Within exact flows (2), the full $R_{k}$-dependence has been studied in [30] by evaluating the fixed points of (2) for general cutoffs (Fig. 1, first column). The main result is that the range of achievable values is bounded from above and from below. The upper bound is attained for Callan-Symanzik type flows with $R_{k} \sim k^{2}$. The lower bound with $\nu=\nu_{\text {opt }}$ is attained with the optimal flow (3), and hence equivalent to the Wilson-Polchinski flow. The sharp cutoff result is indicated for comparison.

The proper-time flow (5) rests on an intrinsically different truncation, because implicit dependences on the background field have been neglected as well as higher order flow terms proportional to the flow of $\Gamma^{(2)}$; see Sec. III. Therefore scheme dependences are quantitatively differ-

\footnotetext{
${ }^{1}$ The variance of the Gaussian measure in (8) can be changed by an explicit rescaling of the fields as $\varphi \rightarrow \varphi / \sqrt{\sigma}$ for finite $\sigma$, see [19]. Rescaling also the integration variable $\xi \rightarrow \xi / \sqrt{\sigma}$, and denoting the potential in terms of the rescaled fields again as $v(\varphi)$, we obtain (8) with a rescaled measure $\mu_{\ell}(\xi)=$ $N_{\ell} \sigma^{-1 / 2} \exp \left(-\xi^{2} / \sigma\right)$. It agrees with the measure of Dyson's model for $N_{\ell}=\pi^{-1 / 2}$ and $\sigma=\sigma(\ell)$. I thank Y. Meurice for Email correspondence on this point.
} 


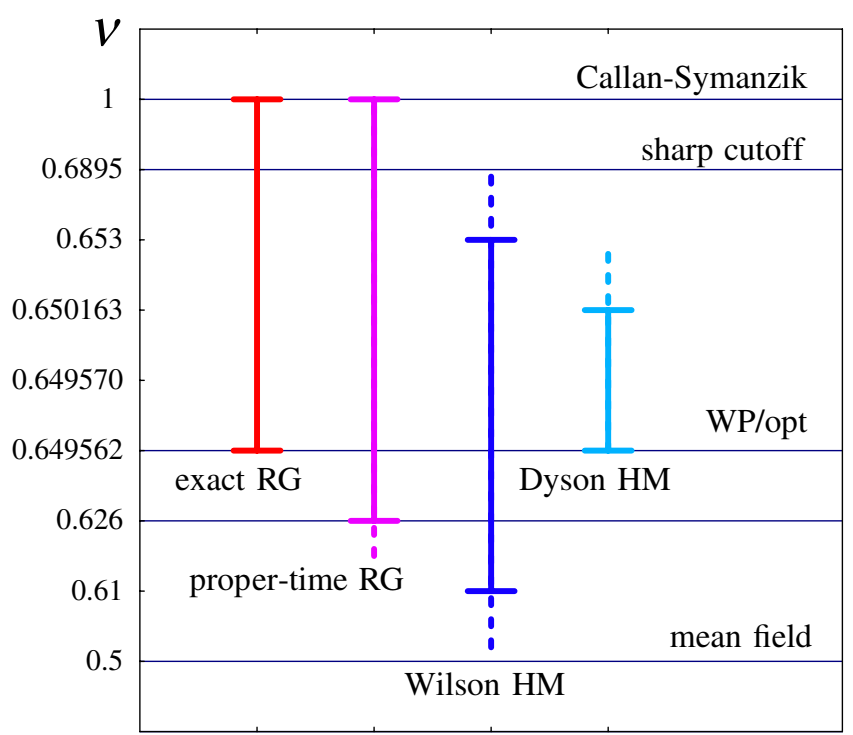

FIG. 1 (color online). Comparison of scaling exponent $\nu$ from different functional flows $(\mathrm{RG})$ in the local potential approximation, and hierarchical models (HM). The solid lines indicate the range of values obtained in the literature. The dashed lines, if present, indicate that the underlying parameter space has not been exhausted. The horizontal lines from top to bottom indicate the results for Callan-Symanzik flows, the sharp cutoff flow, the Wilson-Polchinski (optimal) flow, and the mean field result. A nonlinear rescaling of the $\nu$-axis is introduced for display purposes only (see main text). Color coding: exact RG (red), exact background field $\mathrm{RG}$ in the proper-time approximation (violet), Wilson's hierarchical model (blue) and Dyson's hierarchical model (light blue).

ent. In the approximation (4), the $m$-dependence of scaling exponents from (5) has been studied in [39,42] (Fig. 1, second column). The range of values is again bounded from above by a Callan-Symanzik flow. The lower bound is achieved for $m \rightarrow \infty$. We note that the range of values exceeds those achievable within (standard) exact flows. The lower bound may be overcome once the additional flow terms, neglected here, are taken into account [39]. This is indicated by the dashed line.

Next we consider scheme dependences of hierarchical models. Based on their construction, we expect that physical observables depend on the averaging procedure, on the measure factor $\mu_{\ell}$, and on the decimation parameter $\ell$. It has proven difficult to systematically include wave function renormalizations and higher order operators in hierarchical models, and it is therefore not known whether the scheme dependence vanishes upon higher order corrections $[18,19]$. Still, the scheme dependence should give a reasonable estimate for the underlying error in the model assumptions, in particular, in comparison with functional methods.

The $\ell$-dependence of Wilson's model (8), originally constructed for $\ell \approx 2$, has been studied in [44] in the range $\ell \in\left[2^{1 / 3}, 2\right]$ (Fig. 1, third column). The full line covers the range of values obtained in the literature, while the dashed lines indicate that the underlying parameter space has not been exhausted. Because of (9) being linear in the potential as opposed to (7), we expect a strong $\ell$-dependence, possibly a discontinuity, in the limit $\ell \rightarrow 1$. The slope $\ell \partial_{\ell} \nu(\ell)$ along the data points with $\ell>2^{1 / 3}$ is negative, meaning that $\nu(\ell)$ increases for smaller $\ell$. We stress that Wilson's HM has an overlap both with exact flows and proper-time flows. Therefore, it is possible to map $\nu(\ell)$ of Wilson's model for certain decimation parameters $\ell$ onto $\nu(R)$ from functional flows with appropriately chosen $R$. On the other hand, for some decimation parameter $\ell$, Wilson's model can only be mapped onto proper-time flows but not on exact flows, while for some decimation parameter it cannot be mapped onto either of them.

The $\ell$-dependence of Dyson's model (6) is displayed in Fig. 1, fourth column. The full line connects the known results at $\ell=1[28,30], \ell=2^{1 / 3}[20,22]$ and $\ell=2$ [20]. The dashed line towards larger values for $\nu$ indicates that the parameter space $\ell \geq 1$ has not been exhausted. We note that the $\ell$-dependence is very weak, with a tiny slope in the range of $\ell$-values covered. The important observation is that the slope $\ell \partial_{\ell} \nu(\ell)$ is positive in the vicinity of $\ell \approx$ $1-2$, implying that $\nu(\ell)>\nu(1)$ for $\ell>1$. Consequently, it is possible to map the scaling exponent $\nu(\ell)$ at discrete block-spin transformation $\ell>1$ onto $\nu(R)$ from functional flows for specific momentum cutoff $R$, both within the standard exact flows and within proper-time flows. This supports the conjecture that Dyson's model can be mapped onto functional flows.

\section{SPECTRUM OF EIGENVALUES}

Whether the observations of the preceding section can be promoted to a full map between the formalisms crucially depends on further observables including the subleading scaling exponents. Here and in the following section, we study the spectrum of universal eigenvalues (scaling exponents) form functional flows (2) to high accuracy. A fixed point solution $u_{*} \neq$ const. of (2) is characterized by the universal eigenvalues of eigenperturbations in its vicinity. We denote the ordered set of eigenvalues as $\mathcal{O}(R)=\left\{\omega_{i}(R), i=0, \cdots, \infty\right\}$, with $\omega_{i}<\omega_{j}$ for $i<j{ }^{2}$ In addition to the leading exponent $\nu(R) \equiv$ $-1 / \omega_{0}$, we study the first three subleading scaling exponents $\omega(R) \equiv \omega_{1}(R), \omega_{2}(R)$ and $\omega_{3}(R)$ within the exact flow (2) for various cutoffs and coarse graining parameters.

For the numerical analysis, we introduce several classes of momentum cutoffs defined through $r_{\operatorname{mexp}}=b /((b+$ $\left.1)^{y}-1\right), r_{\exp }=1 /\left(\exp c y^{b}-1\right) ; r_{\text {mod }}=1 /(\exp [c(y+(b-$ 1) $\left.\left.\left.y^{b}\right) / b\right]-1\right)$, with $c=\ln 2$; and $r_{\mathrm{opt}, n}=b(1 / y-1)^{n} \theta(1-$ $y)$. These cutoffs include the sharp cutoff $(b \rightarrow \infty)$ and

\footnotetext{
${ }^{2}$ In our conventions, the sole negative eigenvalue at the Wilson-Fisher fixed point is $\omega_{0}$.
} 
asymptotically smooth Callan-Symanzik type cutoffs $R_{k} \sim$ $k^{2}$ as limiting cases. The larger the parameter $b$, for each class, the "sharper" the corresponding momentum cutoff. The cutoff $r_{\text {opt } n}$ probes a two-dimensional parameter space in the vicinity of $r_{\text {opt }}$ to which it reduces for $b=1$ and $n=$ 1. For integer $n, r_{\mathrm{opt}, n}$ is a $C^{(n+1)}$ function. In addition, we consider the cutoffs $r_{\text {mix }}=\exp [-b(\sqrt{y}-1 / \sqrt{y})]$ and $r_{\text {mix }, \text { opt }}=\exp \left[-\frac{1}{\mathrm{~b}}\left(y^{b}-y^{-b}\right)\right]$, which obey $r_{\text {mix }}(1 / y)=$ $1 / r_{\text {mix }}(y)$. Note that we have covered a large variety of qualitatively different momentum cutoffs including exponential, algebraic, power-law, sharp cutoffs and cutoffs with compact support. Except for $r_{\mathrm{opt}, n}$, all cutoffs are $C^{(\infty)}$-functions. We employ the numerical techniques developed in $[28,30]$.
Our results for the universal eigenvalues at criticality are displayed in Fig. 2 for the six two-dimensional projections of the four-dimensional subspace $\left\{-\omega_{0}, \omega, \omega_{2}, \omega_{3}\right\}$ of observables. The plot contains roughly $10^{3}$ data points, the different classes of cutoffs are color-coded. We focus on the relevant $10 \%$-vicinity of the Wilson-Polchinski result with scaling exponents $\mathcal{O}_{\text {opt }} \equiv \mathcal{O}\left(R_{\text {opt }}\right)$ from (3), indicated by a large black dot, see [28] for the high-accuracy numerical values. The central result of Fig. 2 is that scaling exponents are very strongly correlated. Despite having probed the space of observables by many qualitatively different momentum cutoffs, we find that only a small subset of values can actually be achieved. The correlations increase the closer the eigenvalues $\mathcal{O}$ move towards $\mathcal{O}_{\text {opt }}$. In the immediate vicinity of the Wilson-Polchinski result,
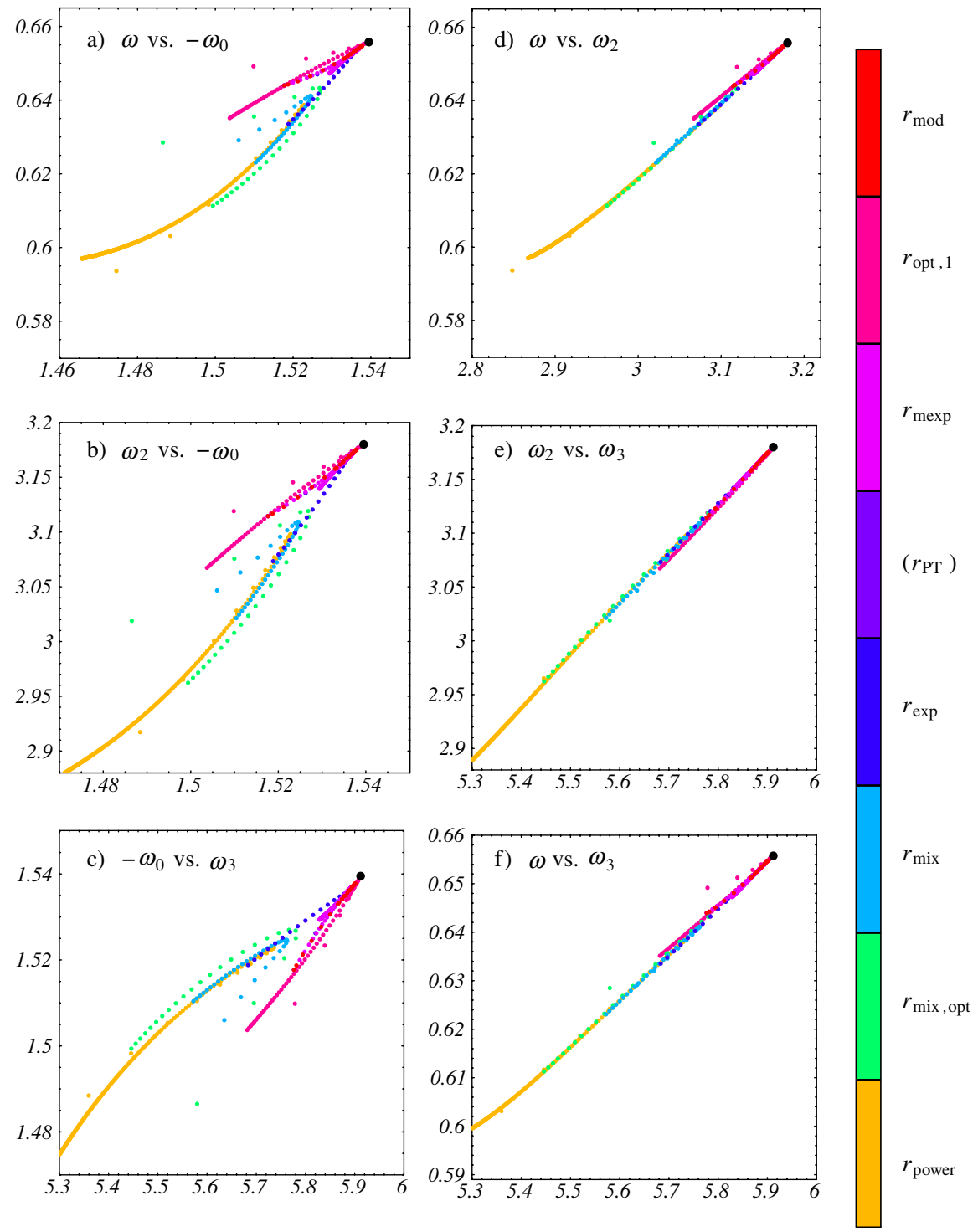

FIG. 2 (color online). Six two-dimensional projections of the four leading scaling exponents in the Ising universality class from the functional flow (2) for various cutoffs and coarse graining (approximately $10^{3}$ data sets). Here, $\nu \equiv-1 / \omega_{0}$. The Wilson-Polchinski result from the optimal flow (3) (large black dot) corresponds to a local extremum for all scaling exponents. Data sets based on $r_{\text {power, }}$, $r_{\text {mix }, \text { opt }}, r_{\text {mix }}, r_{\text {exp }}, r_{\text {mexp }}, r_{\text {opt } 1}$, and $r_{\text {mod }}$; data from $r_{\mathrm{PT}}$ is included in Fig. 4. 
we only find a very narrow "throat" connecting observables $\mathcal{O}(R)$ with $\mathcal{O}_{\text {opt }}$. For the subleading scaling exponents $\omega_{i}$, the throat remains very narrow even further away from $\mathcal{O}_{\text {opt }}$. This is seen most clearly in the correlation of $\omega_{2}$ with $\omega_{3}$ in Fig. 2(e), as well as in the correlations of $\omega$ with both $\omega_{2}$ and $\omega_{3}$ in Fig. 2(d) and 2(f). In turn, for the leading exponents $\nu$, the throat opens up more rapidly once its value is further away from $\nu_{\text {opt }}$, see Fig. 2(a)-2(c).

\section{CORRELATIONS OF EIGENVALUES}

The strong correlation of scaling exponents is a structural fingerprint of Wilsonian flows (2). Since the WilsonPolchinski result is distinguished in the space of scaling exponents, it is natural to normalise the data of Fig. 2 with respect to it. We introduce the distance of any pair of scaling exponents $(x, y)$ from the optimal result $\left(x_{\mathrm{opt}}, y_{\mathrm{opt}}\right)$ as

$$
\rho(x, y)=\sqrt{\left(x_{\mathrm{opt}}-x\right)^{2}+\left(y_{\mathrm{opt}}-y\right)^{2}} \equiv 10^{-N_{\rho}(x, y)} .
$$

We have chosen a standard metric in the space of observables (other choices can be applied as well). In this representation, full agreement with the (optimal) WilsonPolchinski result is achieved for $\rho \rightarrow 0$ and $N_{\rho} \rightarrow \infty$. We also introduce the angles

$$
\varphi(x, y)=\arctan (x / y) .
$$

The critical indices $\nu$ and $\omega_{i}, i \geq 1$, are positive numbers. Therefore, they can cover the range $x / y \in[0, \infty]$ and $\varphi \in$ $\left[0, \frac{\pi}{2}\right]$, and $\rho \geq 0$ for any pair of observables $(x, y)$. In the subspace $(\nu, \omega)$, the extremal values $\left(\nu_{\text {opt }}, \omega_{\text {opt }}\right)$ have the polar coordinates $\left(\rho_{\text {opt }}, \varphi_{\text {opt }}\right)$, where the angle reads $\varphi_{\text {opt }}=$ $0.78066 \cdots$ which is close to $\pi / 4=0.785398 \cdots$, and $\rho_{\text {opt }}=0$. The radial distance from the origin is $\rho(0,0)=$ $0.923002 \cdots$.

In the representation (10), we can study the close vicinity of the Wilson-Polchinski result. In Fig. 3, we display our data points as functions of the angles $\varphi$, and their distance from $\rho_{\text {opt }}$ in a semilogarithmic basis. It is noteworthy that only a very narrow range of angles $\varphi$ is actually achieved by the data, despite the fact that large fractions of the underlying space of momentum cutoffs is covered. Also, and in contrast to Fig. 2(a), many data points are degenerate in the representation $\left(N_{\rho}, \varphi\right) . A$ priori, the Wilson-Polchinski value $\left(\rho_{\text {opt }}, \varphi_{\text {opt }}\right)$ could have been approached along many different paths. Instead, we find that only a narrow range of $\left(N_{\rho}, \varphi\right)$-values is achieved for arbitrary momentum cutoff.

This pattern is further highlighted in Fig. 4, where we have magnified the nontrivial range of data sets from functional flows (2). In addition, we have added data points from the background field flow (5) using the cutoff $r_{\mathrm{PT}, m}$

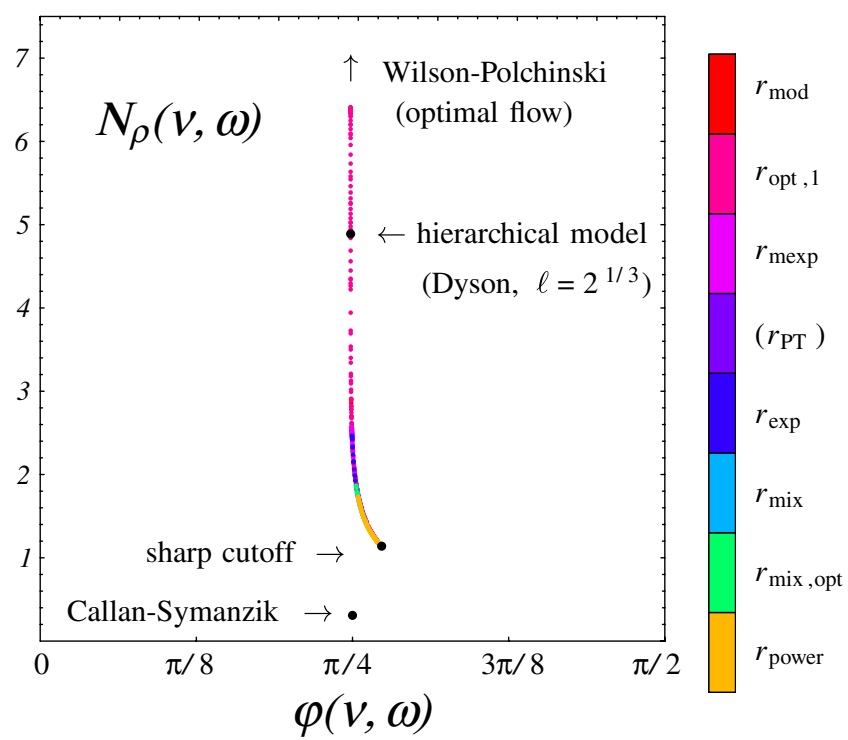

FIG. 3 (color online). Distance $N_{\rho}$ of the pair of scaling exponents $(\nu, \omega)$ from the optimal Wilson-Polchinski values $\left(\nu_{\text {opt }}, \omega_{\text {opt }}\right)$ in the representation (10) and (11). Only a narrow range of angles $\varphi(\nu, \omega)$ in the vicinity of $\varphi \approx \pi / 4$ is achieved by the data. Many data points are nearly degenerate. Data from functional flows in the local potential approximation (2); same data sets and color coding as in Fig. 2, plus further high resolution data points from $r_{\mathrm{opt}, 1}$ in the close vicinity of $\left(\nu_{\text {opt }}, \omega_{\text {opt }}\right)$. Results from the sharp cutoff limit, the CallanSymanzik type flow (with $R_{k} \sim k^{2}$ ) and Dyson's hierarchical model (with $\ell=2^{1 / 3}$ ) are also indicated (black dots). The Wilson-Polchinski (optimal flow) result corresponds to $\varphi=$ $\varphi_{\text {opt }}$ and $N_{\rho} \rightarrow \infty$.

for $m<\frac{5}{2}$. It is remarkable that these data sets display the same pattern as the data from (2). Our results from this and the preceding section are summarized as follows:

Extremum. - The Wilson-Polchinski (optimal flow) result in Fig. 2 corresponds to an extremum in the space of physical observables with $\left|\omega_{i}\right| \leq\left|\omega_{i, \text { opt }}\right|$ for all obervables in the vicinity of $\mathcal{O}_{\text {opt }}$. The extremum is local, because the exponents approach $\omega_{i}=2 i-1, i \geq 0$, for very soft (Callan-Symanzik-type) momentum cutoffs [30]. For the eigenvalue products $\prod_{i=0}^{n}\left(\omega_{i} / \omega_{i, \text { opt }}\right)$, the WilsonPolchinski extremum is a global one.

Uniqueness. - Our result indicates that the correlations of eigenvalues at the Wilson-Pochinski result are strongest, in the sense that any flow of the form (2) with the exponent $\nu(R)=\nu_{\text {opt }}$ automatically also agrees with the WilsonPolchinski result in all other observables $\mathcal{O}(R)=\mathcal{O}_{\text {opt }}$. In general, for $\nu(R)>\nu_{\mathrm{opt}}$, this is clearly not the case.

Redundancy. - The eigenvalue correlations are so strong that the first two scaling exponents $\nu(R)$ and $\omega(R)$, for a given $R$, contain enough information to fix the remaining observables on the percent level or below. These "dynamical" constraints point at a major redundancy of (2) with respect to the underlying momentum cutoffs $R$. A relevant 


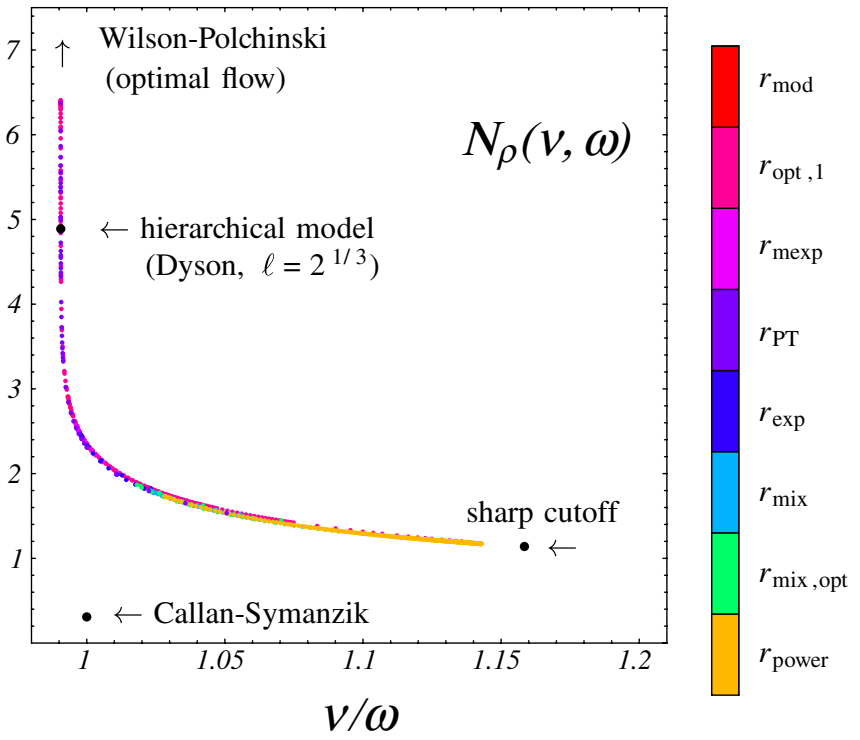

FIG. 4 (color online). Magnification of Fig. 3 in the vicinity of $\varphi \approx \pi / 4$ where $\nu / \omega \approx 1$. The data points for the distance $N_{\rho}(\nu, \omega)$ as a function of $\nu / \omega$ remain highly degenerate. Same data sets and color coding as in Fig. 3, plus additional high resolution data points from background field flows (5) using $r_{\mathrm{PT}, m}$ with $m<\frac{5}{2}$. Results from the sharp cutoff limit, the CallanSymanzik type flow (with $R_{k} \sim k^{2}$ ) and Dyson's hierarchical model (with $\ell=2^{1 / 3}$ ) are also indicated (black dots). The Wilson-Polchinski (optimal flow) result corresponds to $\nu_{\text {opt }} / \omega_{\text {opt }}=0.9905692 \cdots$ and $N_{\rho} \rightarrow \infty$.

parameter has been identified previously. The gap $\min _{y \geq 0} y(1+r)$ for normalized cutoffs [11], when maximized, leads towards the Wilson-Polchinski result [12,13,30].

Optimised observables. - Previous reasonings in favor of an optimization only invoked properties of the underlying flow (1), e.g. its convergence, locality, stability and boundedness, allowing for improved physical predictions. This has been exemplified quantitatively for the observable $\nu(R)$ which obeys $1 \geq \nu(R) \geq \nu_{\text {opt }}$ [30], where the lower bound $\nu_{\text {opt }}$ is closest to the physical result [25]. Figure 2 now shows that this pattern extends to subleading eigenvalues. This equally extends to asymmetric corrections-toscaling [46]. Therefore, one may turn the original reasoning around and argue that-because of the extremum property of the observables $\mathcal{O}(R)$ - an extremization of the functional flow along the lines discussed in $[11,12,14]$, or similar, should naturally lead towards the values $\mathcal{O}_{\text {opt }}$. Stated differently, Figs. 2-4 show that observables derived from (1) admit an optimization.

Finally, we note that the data point from Dyson's hierarchical model with $\ell=2^{1 / 3}$-as plotted in Figs. 3 and 4 - nicely fits into the set of data points covered by functional flows, extending the link observed in Sec. $\mathrm{V}$ beyond the leading exponent. This observation is addressed quantitatively in the following section.

\section{MATCHING BEYOND THE LEADING EXPONENT}

To further substantiate our conjecture that hierarchical models could be mapped onto functional flows, we have to show quantitatively that results from hierarchical model are reproduced by specific functional flows. Here, we study the close vicinity of the Wilson-Polchinski result $\mathcal{O}_{\text {opt }}$, where the correlations are strongest, see Figs. 2 and 3. We have to restrict our search to Dyson's hierarchical model, where high-accuracy data for the first subleading scaling exponent $\omega$ is available. No subleading exponents have been computed for Wilson's model.

For the numerical analysis, we introduce additional classes of momentum cutoffs $R$ which contain the optimal flow (3) in some limit. In addition to the two-parameter family of cutoffs $r_{\mathrm{opt}, n}$, we also study the cutoff $r_{\text {compact }}=$ $y^{-1} \exp \left[-e^{-1 / y} /(b-y)\right] \theta(b-y)$ for $b>0$ which is $C^{(\infty)}$, and the cutoff $r_{\text {int }}=\exp (-y) \theta(1-y) \theta(y-b)$ with $b \in$ $[0,1]$, which is effective for a finite interval of momenta $q^{2} \in\left[b k^{2}, k^{2}\right]$. In the limit $b \rightarrow 0(b \rightarrow 1)$, the corresponding flows are equivalent to (3). Hence, $r_{\mathrm{opt}, n}, r_{\text {compact }}$ and $r_{\text {int }}$ parametrize substantially different classes of cutoffs. More generally, there are infinitely many cutoffs $R_{k}$ leading to scaling exponents identical with $\mathcal{O}_{\text {opt }}$, and the examples provided above serve to illustrate this.

At $\ell=1$, Dyson's hierarchical transformation is continuous, and the scaling exponents are equivalent to those from the optimal flow (3) and the Wilson-Polchinski flow (7). In Table I, we compare exponents from different functional flows. We confirm numerically, and with high accuracy, that the cutoffs $r_{\mathrm{opt}, n}, r_{\text {compact }}$, and $r_{\text {int }}$ lead to the Wilson-Polchinski result for specific parameter values.

At $\ell=2^{1 / 3}$, Dyson's hierarchical transformation is discrete. The reference data reads $\nu_{\mathrm{DHM}}=0.649570$ and $\omega_{\text {DHM }}=0.655736$ [22]..$^{3}$ These values differ only at the order $10^{-5}$ from the optimal (Wilson-Polchinski) result, and are therefore sufficiently close to $\mathcal{O}_{\text {opt }}$ to confirm or refute the correlations observed in the previous section. Figures 3 and 4 indicate that the result from Dyson's hierarchical model is fully matched by functional flows. Our numerical results are given in Table II; brackets indicate that a digit is possibly affected by numerical errors. We have found several sets of parameter values, such that the scaling exponents agree with Dyson's model to order $10^{-6}$. More importantly, the momentum cutoffs are quite different. Hence, our analysis also confirms the strong correlation of scaling exponents in the immediate vicinity of the Wilson-Polchinski result. Based on the eigenvalue correlations within functional flows, we conjecture that the

\footnotetext{
${ }^{3}$ In [22], high-accuracy results at $\ell=2^{1 / 3}$ have been given for $\gamma=2 \nu$ and $\Delta=\nu \omega$ (and $\eta=0$ ) with 13 significant digits. They imply $\nu_{\mathrm{DHM}}=0.649570365 \cdots$ and $\omega_{\mathrm{DHM}}=$ $0.655736286 \cdots$. For the present study, only the first six figures are required.
} 
TABLE I. Matching scaling exponents $\nu$ and $\omega$ from continuous hierarchical transformations with functional flows. Results agree at least to the order $10^{-12}$. Data from this work and from [28].

\begin{tabular}{lclcc}
\hline \hline method & cutoff & parameter & $\nu$ & $\omega$ \\
\hline hierarchical model & Dyson & $(\ell=1)$ & $0.649561773880^{\mathrm{a}}$ & $0.655745939193^{\mathrm{a}}$ \\
\hline \multirow{3}{*}{ functional RG } & $r_{\mathrm{opt}, n}$ & $(n=1, b=1)$ & $0.649561773880^{\mathrm{a}}$ & $0.655745939193^{\mathrm{a}}$ \\
& $r_{\text {compact }}$ & $(b \rightarrow 0)$ & 0.649561773880 & 0.655745939193 \\
& $r_{\text {int }}$ & $(b \rightarrow 1)$ & 0.649561773880 & 0.655745939193 \\
& $r_{\mathrm{PT}, m}$ & $(m=5 / 2)$ & $0.649561773880^{\mathrm{a}}$ & $0.655745939193^{\mathrm{a}}$ \\
\hline \hline
\end{tabular}

${ }^{\mathrm{a}}$ Data from [28].

subleading eigenvalues $\omega_{i}$ with $i \geq 2$ of Dyson's model at $\ell=2^{1 / 3}$ also agree to the corresponding accuracy with the values implied through the functional flows in Table II.

Our results based on the proper-time flow (5) with $r_{\mathrm{PT}}$ has also been given in Table II. Full agreement is achieved either with the exponent $\nu_{\mathrm{DHM}}$ or the subleading exponent $\omega_{\text {DHM }}$, but not with both of them. Once one of them is matched, the deviation in the other observable is of the order $10^{-5}$. The relevant parameters are $m<\frac{5}{2}$, the regime where (5) is mapped onto (2) [39]. Therefore, the values in Table II reflects well the range covered by standard Wilsonian flows (2). We expect that full agreement is achieved for proper-time flows which are linear combinations of (5) for different $m$, but we did not attempt to do so here.

In summary, we have provided numerical maps from several functional flows onto Dyson's model at a nontrivial $\ell \neq 1$, with an accuracy of the order $10^{-6}$. The set of achievable values for scaling exponents from functional flows in the close vicinity of the optimal result is just wide enough to accommodate for the data from Dyson's model. This is a nontrivial result, also showing that the $\ell$-dependence of Dyson's model and the $R_{k}$-dependence of functional flows are very intimately related. Based on our results for $\nu$ and $\omega$ at $\ell \neq 1$, and on continuity in $\ell$, we expect that this map extends to other universal quantities in the same approximation, analogous to the full map which is known for $\ell=1$. Data for further symmetric and asymmetric corrections-to-scaling exponents, once available, will allow for additional checks of this picture. Full equivalence is guaranteed as soon as an explicit link in the form $\ell=\ell\left(R_{k}\right)$ or $R_{k}\left(q^{2}\right)=R_{k}\left(q^{2}, \ell\right)$ is furnished. For the local potential approximation, our results indicate that this map, if it exists, is not unique.

\section{DISCUSSION AND CONCLUSIONS}

Establishing equivalences between implementations of Wilson's renormalization group as different as discrete hierarchical models of lattice scalar fields on one side and continuous functional flows on the other, allows for new views and insights on the respective formalisms and on the underlying physics. Previously, equivalences were known only in the limit where the hierarchical transformation becomes continuous. In this paper, based on similarities in the dependences related to the underlying coarsegraining, we have extended this link towards discrete hierarchical transformations. This correspondence shows that continuous RG flows (1) are sensitive to implicit discretization effects via the momentum cutoff.

Specifically, for the $3 d$ Ising universality class, we have compared the formalisms on the level of scaling exponents.

TABLE II. Matching scaling exponents $\nu$ and $\omega$ from discrete hierarchical transformations with functional flows. Results agree to the order $10^{-6}$ for all cutoffs except the proper-time flow, which matches up to the order $10^{-5}$. Data from this work, and from [22].

\begin{tabular}{lllll}
\hline \hline method & cutoff & parameter & $\nu$ & $\omega$ \\
\hline hierarchical model & Dyson & $\left(\ell=2^{1 / 3}\right)$ & $0.649570^{\mathrm{a}}$ & $0.655736^{\mathrm{a}}$ \\
\hline & $r_{\mathrm{opt}, n}$ & $(n=1, b=1.048)$ & $0.649570(9)$ & $0.655736(6)$ \\
& $r_{\mathrm{opt}, n}$ & $(n=1, b=0.9545)$ & $0.649570(9)$ & $0.655736(9)$ \\
& $r_{\mathrm{opt}, n}$ & $(n=1.135, b=1)$ & $0.649570(6)$ & $0.655736(8)$ \\
functional RG & $r_{\mathrm{opt}, n}$ & $(n=1.1, b=1.028)$ & $0.649570(6)$ & $0.655736(8)$ \\
& $r_{\mathrm{compact}}$ & $(b=0.04775)$ & $0.649570(9)$ & $0.655736(9)$ \\
& $r_{\mathrm{int}}$ & $(b=0.944)$ & $0.649570(9)$ & $0.655736(8)$ \\
& $r_{\mathrm{int}}$ & $(b=0.9444)$ & $0.649570(7)$ & $0.655736(9)$ \\
& $r_{\mathrm{PT}, m}$ & $(m=2.499785)$ & $0.649570(1)$ & $0.655736(1)$ \\
\hline
\end{tabular}

${ }^{\mathrm{a}}$ Data from [22]. 
Their dependence on the step-size parameter $\ell$ within Dyson's hierarchical model (6) is qualitatively and quantitatively similar to their dependence on the momentum cutoff $R$ within functional flows (2). In either case, scaling exponents are bounded by the Wilson-Polchinski values obtained for $\ell \rightarrow 1$ and $R \rightarrow R_{\text {opt }}$. Once the hierarchical transformations are discrete, $\ell \neq 1$, slight variations in all known scaling exponents from Dyson's model are matched by functional flows with nonoptimal momentum cutoffs $R \neq R_{\text {opt }}$. This is quite remarkable, particularly in view of the strong eigenvalue correlations found amongst functional flows. In this light, the optimization of functional flows with $R \rightarrow R_{\text {opt }}$ can now, alternatively, be viewed as the removal of discretization effects, at least to leading order in a derivative expansion as studied here. It will be interesting to contrast these findings with the construction of improved or perfect actions on the lattice. More generally, it is conceivable that Dyson's model for arbitrary $\ell$ is mapped by functional flows on a fundamental level beyond the numerical map provided for $\ell=2^{1 / 3}$. An explicit map would be very welcome, also in view of linking hierarchical models to a path integral representation of the theory. In Wilson's hierarchical model (8), the range covered by the leading scaling exponent indicates that a partial map onto functional flows exists, though only for a restricted domain of $\ell$-values. Interestingly, the overlap with background field flows is even larger. Whether these maps extend beyond the leading exponent cannot be settled presently due to a lack of data for subleading exponents from Wilson's model.

In addition, we found a distinct correlation of scaling exponents from functional flows (2). The eigenvalue spectrum, a fingerprint of the physics in a local potential approximation, is severely constrained and achieves the Wilson-Polchinski values as an extremum. Furthermore, the full space of physical observables is described by very few parameters only, instead of the infinitely many moments of the momentum cutoff. Resolving this redundancy should prove useful for studies of e.g. nontrivial momentum structures and higher orders in the derivative expansion. Finally this pattern amongst physical observables highlights the extremum property of cutoffs leading to the optimal Wilson-Polchinski result [11-14]. We expect that the intimate link between optimized flows on one side, and extremum points in the space of observables on the other, persists in more complex theories. This observation will prove useful for studies in QCD and quantum gravity, where an appropriate optimization is even more important to extract the relevant physics.

\section{ACKNOWLEDGMENTS}

This work is supported by EPSRC. I thank Y. Meurice for email correspondence, the Galileo Galilei Institute for Theoretical Physics for hospitality, and the INFN for partial support.
[1] J. Zinn-Justin, Quantum Field Theory And Critical Phenomena (Clarendon, Oxford, 1989).

[2] K. G. Wilson and J. B. Kogut, Phys. Rep. 12, 75 (1974).

[3] N. Tetradis and D. F. Litim, Nucl. Phys. B464, 492 (1996).

[4] T. R. Morris, Prog. Theor. Phys. Suppl. 131, 395 (1998).

[5] D.F. Litim, in Proceedings of TFT98: Thermal Field Theories and Their Applications, edited by U. Heinz; hep-th/9811272.

[6] D.F. Litim and J.M. Pawlowski, in The Exact Renormalization Group, edited by Krasnitz et al. (World Scientific, Singapore, 1999), p. 168.

[7] C. Bagnuls and C. Bervillier, Phys. Rep. 348, 91 (2001).

[8] J. Berges, N. Tetradis, and C. Wetterich, Phys. Rep. 363, 223 (2002).

[9] J. Polonyi, Central Eur. J. Phys. 1, 1 (2003).

[10] M. Salmhofer and C. Honerkamp, Prog. Theor. Phys. 105, 1 (2001).

[11] D. F. Litim, Phys. Lett. B 486, 92 (2000).

[12] D. F. Litim, Phys. Rev. D 64, 105007 (2001).

[13] D. F. Litim, Int. J. Mod. Phys. A 16, 2081 (2001).

[14] J. M. Pawlowski, arXiv:hep-th/0512261.

[15] F. J. Dyson, Commun. Math. Phys. 12, 91 (1969).

[16] K. G. Wilson, Phys. Rev. B 4, 3184 (1971); Phys. Rev. D 6 , 419 (1972).
[17] G. Baker, Phys. Rev. B 5, 2622 (1972).

[18] G. Golner, Phys. Rev. B 8, 339 (1973).

[19] Y. Meurice, J. Phys. A 40, R39 (2007).

[20] H. Koch and P. Wittwer, in Nonlinear Evolution and Critical Phenomena, edited by G. Gallavotti and P. Zweifel NATO Advanced Study Institute, Vol. 176 (Plenum Press, New York, 1988), p. 269.

[21] K. Pinn, A. Pordt, and C. Wieczerkowski, J. Stat. Phys. 77, 977 (1994); J. J. Godina, Y. Meurice, M. B. Oktay, and S. Niermann, Phys. Rev. D 57, 6326 (1998); J. J. Godina, Y. Meurice, and M.B. Oktay, Phys. Rev. D 59, 096002 (1999); J. Gottker-Schnetmann, arXiv:cond-mat/9909418.

[22] J. J. Godina, Y. Meurice, and M. B. Oktay, Phys. Rev. D 57, R6581 (1998).

[23] J. Polchinski, Nucl. Phys. B231, 269 (1984).

[24] G. Felder, Commun. Math. Phys. 111, 101 (1987).

[25] D. F. Litim, J. High Energy Phys. 07 (2005) 005.

[26] T. R. Morris, J. High Energy Phys. 07 (2005) 027.

[27] C. Wetterich, Phys. Lett. B 301, 90 (1993).

[28] C. Bervillier, A. Jüettner, and D. F. Litim, Nucl. Phys. B783, 213 (2007).

[29] D. F. Litim, J. High Energy Phys. 11 (2001) 059.

[30] D. F. Litim, Nucl. Phys. B631, 128 (2002).

[31] J. M. Pawlowski, D. F. Litim, S. Nedelko, and L. von 
Smekal, Phys. Rev. Lett. 93, 152002 (2004); in Quark Confinement and the Hadron Spectrum VI, edited by N. Brambilla et al., AIP Conf. Proc. No. 756 (AIP, New York, 2005), p. 278; D. F. Litim, J. M. Pawlowski, S. Nedelko, and L. V. Smekal, arXiv:hep-th/0410241.

[32] O. Lauscher and M. Reuter, Classical Quantum Gravity 19, 483 (2002); D. F. Litim, Phys. Rev. Lett. 92, 201301 (2004); R. Percacci, Phys. Rev. D 73, 041501 (2006); P. Fischer and D. F. Litim, Phys. Lett. B 638, 497 (2006); in Albert Einstein Century International Conference, edited by J-M. Alimi and A. Füzfa, AIP Conf. Proc. No. 861 (AIP, New York, 2006), p. 336; A. Codello and R. Percacci, Phys. Rev. Lett. 97, 221301 (2006).

[33] D. F. Litim and J. M. Pawlowski, J. High Energy Phys. 11 (2006) 026; J. P. Blaizot, A. Ipp, R. Mendez-Galain, and N. Wschebor, Nucl. Phys. A784, 376 (2007).

[34] J.P. Blaizot, R. Mendez Galain, and N. Wschebor, Europhys. Lett. 72, 705 (2005).

[35] D. F. Litim and N. Tetradis, arXiv:hep-th/9501042.

[36] M. Reuter and C. Wetterich, Nucl. Phys. B417, 181 (1994); F. Freire, D. F. Litim, and J. M. Pawlowski, Phys. Lett. B 495, 256 (2000).

[37] M. Reuter and C. Wetterich, Phys. Rev. D 56, 7893 (1997);
H. Gies, Phys. Rev. D 66, 025006 (2002).

[38] D. F. Litim and J. M. Pawlowski, J. High Energy Phys. 09 (2002) 049.

[39] D. F. Litim and J. M. Pawlowski, Phys. Lett. B 546, 279 (2002); Phys. Lett. B 516, 197 (2001).

[40] D. F. Litim and J. M. Pawlowski, Phys. Rev. D 66, 025030 (2002); 65, 081701 (2002).

[41] S. B. Liao, Phys. Rev. D 56, 5008 (1997); 53, 2020 (1996).

[42] A. Bonanno and D. Zappala, Phys. Lett. B 504, 181 (2001); M. Mazza and D. Zappala, Phys. Rev. D 64, 105013 (2001).

[43] B. J. Schaefer and H. J. Pirner, Nucl. Phys. A660, 439 (1999); B. J. Schaefer and J. Wambach, arXiv:hep-ph/ 0611191 ; Phys. Rev. D 75, 085015 (2007).

[44] Y. Meurice and G. Ordaz, J. Phys. A 29, L635 (1996).

[45] R. D. Ball, P. E. Haagensen, J. I. Latorre, and E. Moreno, Phys. Lett. B 347, 80 (1995); D. F. Litim, Phys. Lett. B 393, 103 (1997); S. B. Liao, J. Polonyi, and M. Strickland, Nucl. Phys. B567, 493 (2000); F. Freire and D. F. Litim, Phys. Rev. D 64, 045014 (2001); L. Canet, B. Delamotte, D. Mouhanna, and J. Vidal, Phys. Rev. B 68, 064421 (2003).

[46] D. F. Litim and L. Vergara, Phys. Lett. B 581, 263 (2004). 